\title{
Short-time traffic flow prediction based on seasonal gray Fourier model
}

\section{Z. Shen ( $\nabla$ shenzhou.research@gmail.com )}

\section{Research Article}

Keywords:

Posted Date: February 7th, 2022

DOI: https://doi.org/10.21203/rs.3.rs-1334110/v1

License: (9) (i) This work is licensed under a Creative Commons Attribution 4.0 International License. Read Full License 


\title{
Short-time traffic flow prediction based on seasonal gray Fourier model
}

\author{
Zhou Shen*
}

\begin{abstract}
To handle the periodic, nonlinear, and stochastic aspects of short-time traffic flow data, a seasonal gray Fourier model based on the complex Simpson formula is proposed. The seasonal GM $(1,1)$ model is used to optimize the background values first, and then the prediction results are adjusted using the Fourier series method. The new model was applied to the prediction of traffic flow on Whitemud Drive in Canada, and the numerical results indicated that the new model's mean absolute percentage error was 1.54 percent and its fit was 0.996 , which were significantly better than those of the traditional GM $(1,1)$ model, the seasonal GM $(1,1)$ model, and the Fourier optimized seasonal GM $(1,1)$ model.
\end{abstract}

\section{Introduction}

Intelligent transportation systems may maximize the potential of current transportation infrastructure, which is a significant step toward increasing transportation efficiency, ensuring traffic safety, and reducing congestion. Short-term traffic flow prediction, as the foundation for traffic guidance and management, is at the heart of intelligent transportation systems and the focus of traffic flow theory research.

Numerous experts and researchers have undertaken study on short-term traffic flow prediction using the various features of short-term traffic flow, such as delay, nonlinearity, volatility, periodicity, and geographical and temporal dependence. For example, Kang Jun proposed a method for predicting short-term traffic flow based on Gaussian process regression, Li Song proposed a method for predicting short-term traffic flow based on an improved particle swarm algorithm optimized BP neural network, Liu Zhao proposed a combined prediction model, and KUMAR proposed a SARIMA model based on the characteristics of short-term traffic flow periodic fluctuations.

Gray prediction has been widely utilized in short-term traffic flow forecasting because to its advantages of requiring less modeling data, achieving a greater prediction accuracy, and having a broad application range. However, due to the complexity of the volatility and nonlinearity of short-term traffic flow, as

\footnotetext{
*The authors declare no competing interests.
} 
well as its seasonal characteristics, the standard GM $(1,1)$ prediction is not very accurate. Numerous scholars have improved the model from a variety of angles, including Guo Huan et alintroduction .'s of the GM $(1,1)$ model, LIU et alestablishment .'s of a rolling GM $(1,1)$ prediction model, Tan Champion et alimprovement .'s of the GM $(1,1)$ model's prediction accuracy through reconstruction of the background values, and HSU et alimprovement .'s of the prediction residuals through the use of Fourier series. Recently, Xiao et al. exploited the seasonal characteristics of short-time traffic flow by first employing a circular truncated cumulative generation operator to reduce the volatility of the data and then proposing a seasonal GM $(1,1)$ (SGM $(1,1)$ ) model based on the original GM $(1,1)$ concept.

To further increase the prediction accuracy of the SGM $(1,1)$ model, this research proposes a new seasonal gray Fourier model based on the complex Simpson formula. The new model optimizes the background values using the complex Simpson formula and corrects the anticipated residuals using the Fourier series method. The anticipated numerical findings indicate that the new model has a lower mean absolute percentage error (MAPE) and a higher goodness of fit (FD) than the conventional GM $(1,1)$ model, the SGM $(1,1)$ model, and the Fourier-optimized SGM $(1,1)$ model.

\section{GM $(1,1)$ Model and SGM $(1,1)$ Model}

\section{1 $\operatorname{GM}(1,1)$ Model}

A set of non-negative primitive series is known as

$$
x^{(0)}=\left(x^{(0)}(1), x^{(0)}(2), \cdots, x^{(0)}(n)\right)
$$

whose once-accumulated (1-AGO) sequence is defined as

$$
x^{(1)}=\left(x^{(1)}(1), x^{(1)}(2), \cdots, x^{(1)}(n)\right)
$$

where,

$$
x^{(1)}(k)=\sum_{i=1}^{k} x^{(0)}(i)
$$

and $\mathrm{k}=1,2, \ldots, \mathrm{n}$. The basic form of the GM $(1,1)$ model is

$$
x^{(0)}(k)+a z^{(1)}(k)=b
$$

where the background values $z^{(1)}(k), \mathrm{k}=2, \ldots, \mathrm{n}$ can be represented by a mean generating sequence, $z^{(1)}(k)=0.5 \mathrm{x}(1)(\mathrm{k})+0.5 x^{(1)}(k-1)$. Let

$$
\boldsymbol{p}=\left[\begin{array}{l}
a \\
b
\end{array}\right], \boldsymbol{Y}=\left[\begin{array}{c}
x^{(0)}(2) \\
x^{(0)}(3) \\
\vdots \\
x^{(0)}(n)
\end{array}\right], \boldsymbol{B}=\left[\begin{array}{cc}
-z^{(1)}(2) & 1 \\
-z^{(1)}(3) & 1 \\
\vdots & \vdots \\
-z^{(1)}(n) & 1
\end{array}\right]
$$


The parameters a, b are obtained by the least squares method,

$$
\boldsymbol{p}=\left(\boldsymbol{B}^{\mathrm{T}} \boldsymbol{B}\right)^{-1} \boldsymbol{B}^{\mathrm{T}} \boldsymbol{Y} .
$$

The corresponding whitening differential equation for the $\operatorname{GM}(1,1)$ model is defined as

$$
\frac{\mathrm{d} x^{(1)}(k)}{\mathrm{d} t}+a x^{(1)}(k)=b
$$

Taking $x^{(0)}(1)$ as the initial condition, the time response series is obtained by solving the above whitening differential equation

$$
\begin{gathered}
\hat{x}^{(1)}(k+1)=\left(x^{(0)}(1)-b / a\right) \mathrm{e}^{-a k}+b / a \\
k=1,2, \cdots, n .
\end{gathered}
$$

The predicted values of the original series are obtained by first order cumulative reduction

$$
\hat{x}^{(0)}(k+1)=\hat{x}^{(1)}(k+1)-\hat{x}^{(1)}(k) .
$$

\section{2 $\operatorname{SGM}(1,1)$ Model}

For the original non-negative sequence

$$
x^{(0)}=\left(x^{(0)}(1), x^{(0)}(2), \cdots, x^{(0)}(n)\right)
$$

Take $\mathrm{q}$ as a cycle, and use the cycle truncation accumulation generation operator (CTAGO) to obtain the new sequence

$$
\begin{gathered}
y^{(0)}=\left(y^{(0)}(1), y^{(0)}(2), \cdots, y^{(0)}(r)\right) \\
r=1,2, \cdots, n-q+1
\end{gathered}
$$

where

$$
\begin{gathered}
y^{(0)}(k)=\mathrm{CTAGO}\left(x^{(0)}(k)\right)= \\
\sum_{j=1}^{q} x^{(0)}(k+j-1), \forall k=1,2, \cdots, n-q+1 .
\end{gathered}
$$

The 1-AGO sequence of $y(0)$ is

$$
y^{(1)}=\left(y^{(1)}(1), y^{(1)}(2), \cdots, y^{(1)}(r)\right)
$$

The calculation gives

$$
y^{(1)}(k)=\sum_{i=1}^{k} y^{(0)}(i)=\sum_{i=1}^{k} \sum_{j=1}^{q} x^{(0)}(i+j-1) .
$$


Substituting the obtained y $(0)$ sequence into the conventional GM $(1,1)$ model, the basic form of SGM $(1,1)$ model can be defined as

$$
y^{(0)}(k)+a z^{(1)}(k)=b
$$

Let

$$
\boldsymbol{Y}=\left[\begin{array}{c}
y^{(0)}(2) \\
y^{(0)}(3) \\
\vdots \\
y^{(0)}(r)
\end{array}\right], \boldsymbol{B}=\left[\begin{array}{ccc}
-z^{(1)} & (2) & 1 \\
-z^{(1)} & (3) & 1 \\
\vdots & & \vdots \\
-z^{(1)}(r) & 1 &
\end{array}\right]
$$

Similarly, the least squares solution gives

$$
\boldsymbol{p}=(a, b)^{\mathrm{T}}=\left(\boldsymbol{B}^{\mathrm{T}} \boldsymbol{B}\right)^{-1} \boldsymbol{B}^{\mathrm{T}} \boldsymbol{Y} .
$$

The time response sequence of SGM $(1,1)$ model is obtained by solving the whitening differential equation with reference to the GM $(1,1)$ model

$$
\begin{gathered}
\hat{y}^{(1)}(k+1)=\left(y^{(0)}(1)-\frac{b}{a}\right) \mathrm{e}^{-a k}+\frac{b}{a}, \\
k=1,2, \cdots, r,
\end{gathered}
$$

A single cumulative reduction is performed to obtain

$$
\begin{gathered}
\hat{y}^{(0)}(k+1)=\hat{y}^{(1)}(k+1)-\hat{y}^{(1)}(k), \\
k=1,2, \cdots, r,
\end{gathered}
$$

The reduced value is

$$
\begin{gathered}
\hat{x}^{(0)}(k+1)= \\
\hat{y}^{(0)}(k-q+2)-\hat{y}^{(0)}(k-q+1)+x^{(0)}(k-q+1)= \\
\left.\hat{y}^{(1)}(k-q+2)-\hat{y}^{(1)}(k-q+1)\right)- \\
\hat{y}^{(0)}(k-q+1)+x^{(0)}(k-q+1), \\
k=q, q+1, \cdots, n .
\end{gathered}
$$

\section{Seasonal gray Fourier model based on the com- plex Simpson formula}

The SGM $(1,1)$ model firstly accumulates the original data periodically to weaken the volatility of the data, but the new series generated after accumulation is still using the traditional gray forecasting method with limited improvement in accuracy. In this paper, we introduce a new seasonal gray Fourier model based on the complex Simpson formula based on the SGM $(1,1)$ model to further improve the forecasting accuracy. 


\subsection{Improved background values by compounding Simp- son's formula}

The error of the traditional GM $(1,1)$ model is mainly due to the use of the trapezoidal formula

$$
\frac{1}{2}\left[x^{(1)}(k)+x^{(1)}(k-1)\right]
$$

instead of the integral value in the calculation of the background value

$$
\int_{k-1}^{k} x^{(0)}(t) \mathrm{d} t
$$

In order to reduce the error of calculating the background value, this paper uses the complexified Simpson formula to calculate the background value $\mathrm{z}(1)(\mathrm{k})$.

Definition 1: Let $[\mathrm{k}-1, \mathrm{k}]=[\mathrm{a}, \mathrm{b}]$, and divides the interval $[\mathrm{a}, \mathrm{b}$ into $\mathrm{n}$ equal parts, each equal part being

$$
x_{i+\frac{1}{2}}=x_{i}+0.5 h
$$

$\mathrm{h}=(\mathrm{b}-\mathrm{a}) / \mathrm{n}$, then, $\mathrm{i}=0,1, \ldots, \mathrm{n}$, and uses Simpson's formula on each small interval [xi, xi +1 ], thus obtaining the complexified Simpson's formula

$$
\begin{gathered}
S_{n}=\int_{b}^{a} f(x) \mathrm{d} x= \\
\frac{h}{6} \sum_{i=0}^{n-1}\left[f\left(x_{i}\right)+4 f\left(x_{i+\frac{1}{2}}\right)+f\left(x_{i+1}\right)\right]= \\
\frac{h}{6}\left[f(a)+4 \sum_{i=0}^{n-1} f\left(x_{i+\frac{1}{2}}\right)+2 \sum_{i=0}^{n-1} f\left(x_{i}\right)+f(b)\right] .
\end{gathered}
$$

Since 1-AGO is a discrete sequence, it is impossible to find the function value corresponding to xi in the complex Simpson formula, so this paper proposes to use the Lagrangian interpolation to fit the function to obtain

$$
\begin{aligned}
& f\left(x_{i+\frac{1}{2}}\right) \\
& f\left(x_{i}\right)
\end{aligned}
$$

Definition 2: Denote three points $\mathrm{k}-1, \mathrm{k}, \mathrm{k}+1$ as interpolation nodes, and the quadratic interpolation polynomial $\mathrm{f}(\mathrm{t})$ of $\mathrm{x}(1)(\mathrm{t})$ is obtained as

$$
\begin{aligned}
f(t)= & \frac{\left(t-x_{1}\right)\left(t-x_{2}\right)}{\left(x_{0}-x_{1}\right)\left(x_{0}-x_{2}\right)} \cdot x^{(1)}(k-1)+ \\
& \frac{\left(t-x_{0}\right)\left(t-x_{2}\right)}{\left(x_{1}-x_{0}\right)\left(x_{1}-x_{2}\right)} \cdot x^{(1)}(k)+ \\
& \frac{\left(t-x_{0}\right)\left(t-x_{1}\right)}{\left(x_{2}-x_{0}\right)\left(x_{2}-x_{1}\right)} \cdot x^{(1)}(k+1),
\end{aligned}
$$


where $\mathrm{x} 0=\mathrm{k}-1, \mathrm{x} 1=\mathrm{k}, \mathrm{x} 2=\mathrm{k}+1$.

The background value is obtained by substituting the complexified Simpson formula

$$
\begin{aligned}
& z^{(1)}(k)=\int_{k-1}^{k} x^{(1)}(t) \mathrm{d} t= \\
& \frac{h}{6}\left[f(a)+4 \sum_{i=0}^{n-1} f\left(x_{i+\frac{1}{2}}\right)+2 \sum_{i=0}^{n-1} f\left(x_{i}\right)+f(b)\right] .
\end{aligned}
$$

\subsection{Fourier series improved residuals}

Due to the limitation of the traditional gray prediction model that generates a single exponential rate of change cumulatively, its residuals have both positive and negative and are highly volatile. In order to further improve the prediction accuracy of the model, this paper introduces Fourier series to correct the residuals. First, based on the results obtained from the above prediction, the residual series can be obtained

$$
\varepsilon^{(0)}=\left(\varepsilon^{(0)}(2), \varepsilon^{(0)}(3), \cdots, \varepsilon^{(0)}(n)\right)
$$

where

$$
\varepsilon^{(0)}(k)=x^{(0)}(k)-\hat{x}^{(0)}(k), k=2,3, \cdots, n
$$

Let the residuals after the Fourier series correction be

$$
\begin{gathered}
\varepsilon^{\prime(0)}(k)=\frac{1}{2} a_{0}+\sum_{i=1}^{m}\left[a_{i} \cos \left(\frac{2 \pi i}{T} k\right)+b_{i} \sin \left(\frac{2 \pi i}{T} k\right)\right], \\
k=2,3, \cdots, n,
\end{gathered}
$$

where $\mathrm{T}=\mathrm{n}-1, m=\left[\frac{n-1}{2}\right]-1, \mathrm{a} 0, \mathrm{ai}, \mathrm{bi}, \mathrm{i}=1,2, \ldots, \mathrm{m}$ are the Fourier parameters to be found. Let $\epsilon(0)=\mathrm{DC}$, expand as

$$
\left[\begin{array}{c}
\varepsilon^{(0)}(2) \\
\varepsilon^{(0)}(3) \\
\vdots \\
\varepsilon^{(0)}(n)
\end{array}\right]=\left[\begin{array}{cccccc}
\frac{1}{2} & \cos \left(2 \frac{2 \pi}{T}\right) & \sin \left(2 \frac{2 \pi}{T}\right) & \cdots & \cos \left(2 \frac{2 \pi m}{T}\right) & \sin \left(2 \frac{2 \pi m}{T}\right) \\
\frac{1}{2} & \cos \left(3 \frac{2 \pi}{T}\right) & \sin \left(3 \frac{2 \pi}{T}\right) & \cdots & \cos \left(3 \frac{2 \pi m}{T}\right) & \sin \left(3 \frac{2 \pi m}{T}\right) \\
\vdots & \vdots & \vdots & \vdots & \vdots & \vdots \\
\frac{1}{2} & \cos \left(n \frac{2 \pi}{T}\right) & \sin \left(n \frac{2 \pi}{T}\right) & \cdots & \cos \left(n \frac{2 \pi m}{T}\right) & \sin \left(n \frac{2 \pi m}{T}\right)
\end{array}\right]
$$

Using the least squares method to find the parameters

$$
\boldsymbol{C}=\left(\boldsymbol{D}^{\mathrm{T}} \boldsymbol{D}\right)^{-1} \boldsymbol{D}^{\mathrm{T}} \varepsilon^{(0)}
$$

The initial prediction value is corrected by Fourier series to obtain the final prediction value

$$
\left\{\begin{array}{l}
x^{(0)}(1)=\hat{x}^{(0)}(1) \\
\bar{x}^{(0)}(k)=\hat{x}^{(0)}(k)+\varepsilon^{(0)}(k), k=2,3, \cdots, n
\end{array}\right.
$$




\subsection{Model steps}

In summary, the steps of the seasonal gray Fourier model based on the complex Simpson formula are as follows:

Step 1 Determine the original series $\mathrm{x}(0)=(\mathrm{x}(0)(1), \mathrm{x}(0)(2), \ldots, \mathrm{x}$ (0) (n) ), and seasonally accumulate the original series with $q$ as the period to obtain the CTAGO series y (0) (r) , r =1, 2, .., n-q+1;

Step 2 Perform first-order accumulation on the series y (0) (r), construct the background values using the complex Simpson formula, and solve for the parameters a, b;

Step 3 Substitute the parameters into the SGM $(1,1)$ model to obtain the time response series, and calculate the initial prediction value;

Step 4 Calculate the residual $\epsilon(0)(\mathrm{k})$ of the initial prediction and correct it by Fourier series to obtain the final prediction.

\section{Case studies}

\subsection{Data sources}

The traffic flow data used in this paper were obtained from the OpenITS data platform, which provides traffic flow data for the Whitemud Drive highway in Edmonton, Alberta, Canada, from August 8 to 28, 2015, from 7:00 to 8:00 with a time interval of $15 \mathrm{~min}$, and the data were organized to obtain The raw data are shown in Figure 1.

\subsection{Prediction error index}

In order to verify the prediction accuracy and validity of the model, we define the indexes of prediction error - mean absolute percentage error (MAPE) and goodness of fit (FD) The smaller the value of MAPE, the higher the prediction accuracy, i.e., MAPE values below 10\% indicate high accuracy, $11 \%-20 \%$ indicate high accuracy, $21 \%-50 \%$ indicate fair accuracy, and greater than or equal to $51 \%$ indicate poor accuracy. $\mathrm{FD}>0.95$ is considered as a satisfactory prediction.

\subsection{Forecast results}

In this paper, Matlab simulations were performed with the traditional GM (1, 1) model, SGM $(1,1)$ model, Fourier-optimized SGM $(1,1)$ model and the new model for the section measured by the VDS1030 ground sensor coil in the west direction of the highway. In the complex Simpson formula, theoretically, the larger the value of $n$, the more accurate the numerical integration, the higher the fitting and prediction accuracy, but the improvement will be smaller and smaller. In this paper, we take $n=500$ and select the data set of 7:30-7:45 as the object of analysis, and calculate the fitting results as shown in Table 1. 


\begin{tabular}{|c|c|c|c|c|c|c|c|c|c|}
\hline \multirow{2}{*}{ Date } & \multirow{2}{*}{ Ground Truth } & \multicolumn{2}{|c|}{$\operatorname{GM}(1,1)$} & \multicolumn{2}{|c|}{$\operatorname{SGM}(1,1)$} & \multirow{2}{*}{$\begin{array}{l}\text { SGM }(1,1) \\
\text { Predicted }\end{array}$} & Fourier & \multirow{2}{*}{$\begin{array}{l}\text { Proposed } \\
\text { Predicted }\end{array}$} & \multirow[b]{2}{*}{ MAPE } \\
\hline & & Predicted & MAPE & Predicted & MAPE & & MAPE & & \\
\hline 20150808 & 356 & 356.0000 & 0 & 356.0000 & 0 & 356.0000 & 0 & 356.0000 & 0 \\
\hline 20150809 & 179 & 912.8140 & 4.0995 & 179.0000 & 0 & 179.0000 & 0 & 179.0000 & 0 \\
\hline 20150810 & 1326 & 925.7596 & 0.3018 & 1326.0000 & 0 & 1326.0000 & 0 & 1326.0000 & 0 \\
\hline 20150811 & 1327 & 938.8889 & 0.2925 & 1327.0000 & 0 & 1327.0000 & 0 & 1327.0000 & 0 \\
\hline 20150812 & 1364 & 952.2043 & 0.3019 & 1364.0000 & 0 & 1364.0000 & 0 & 1364.0000 & 0 \\
\hline 20150813 & 1381 & 965.7086 & 0.3007 & 1381.0000 & 0 & 1381.0000 & 0 & 1381.0000 & 0 \\
\hline 20150814 & 1242 & 979.4044 & 0.2114 & 1242.0000 & 0 & 1242.0000 & 0 & 1242.0000 & 0 \\
\hline 20150815 & 285 & 993.2945 & 2.4852 & 187.3438 & 0.3427 & 290.8710 & 0.0206 & 280.4784 & 0.0159 \\
\hline 20150816 & 155 & 1007.3815 & 5.4992 & 179.8588 & 0.1604 & 145.2585 & 0.0628 & 164.0432 & 0.0583 \\
\hline 20150817 & 1313 & 1021.6683 & 0.2219 & 1326.8589 & 0.0106 & 1317.9740 & 0.0038 & 1303.9568 & 0.0069 \\
\hline 20150818 & 1287 & 1036.1577 & 0.1949 & 1327.8590 & 0.0317 & 1287.7787 & 0.0006 & 1296.0432 & 0.0070 \\
\hline 20150819 & 1304 & 1050.8527 & 0.1941 & 1364.8591 & 0.0467 & 1297.6228 & 0.0049 & 1294.9568 & 0.0069 \\
\hline 20150820 & 1337 & 1065.7560 & 0.2029 & 1381.8592 & 0.0336 & 1347.7125 & 0.0080 & 1346.0432 & 0.0068 \\
\hline 20150821 & 1237 & 1080.8707 & 0.1262 & 1242.8593 & 0.0047 & 1224.0739 & 0.0104 & 1227.9568 & 0.0073 \\
\hline
\end{tabular}

From Table 1, we can see that the mean values of the conventional GM (1, 1) model, SGM $(1,1)$ model, Fourier-optimized SGM $(1,1)$ model, Simpsonoptimized SGM $(1,1)$ model and the new model MAPE are $109.25 \%, 11.50 \%$, $11.18 \%, 5.76 \%$ and $1.54 \%$, respectively, and the FD values are The results show that the new model outperforms the traditional GM $(1,1)$ model, SGM (1, 1) model, Fourier-optimized SGM $(1,1)$ model and Simpson-optimized SGM $(1,1)$ model in terms of prediction accuracy, and can be effectively applied to short-time traffic flow prediction.

\section{Conclusion}

Accurate traffic flow prediction is a prerequisite for implementing traffic signal control, traffic assignment, path guidance, and accident detection in intelligent transportation systems, and the results of traffic flow prediction will directly affect the effectiveness of traffic control and management. In this paper, we propose a seasonal gray Fourier model based on the seasonal gray prediction model, which makes full use of the periodic, nonlinear and stochastic characteristics of short-time traffic flow data. Compared with the traditional GM $(1,1)$ model and SGM $(1,1)$ model, the new model has obvious accuracy advantage and better fit, which is worth to be applied in practice.

\section{References}

[1] J. J. Q. Yu, "Travel mode identification with GPS trajectories using wavelet transform and deep learning," IEEE Transactions on Intelligent Transportation Systems, vol. 22, no. 2, pp. 1093-1103, 2021.

[2] J. J. Q. Yu et al., "Optimal V2G scheduling of electric vehicles and unit commitment using chemical reaction optimization," in Proc. IEEE Congress of Evolutionary Computation, Cancun, Mexico, 2013, pp. 392-399.

[3] Y. Yan et al., "Multi-objective design optimization of combined cooling, heating and power system for cruise ship application," Journal of Cleaner Production, vol. 233, pp. 264-279, Oct. 2019 .

[4] J. J. Q. Yu et al., "Adaptive chemical reaction optimization for global numerical optimization," in Proc. IEEE Congress of Evolutionary Computation, Sendai, Japan, 2015, pp. 3192-3199. 
[5] Y. Liu et al., "PPGAN: privacy-preserving generative adversarial network," in Proc. IEEE International Conference on Parallel and Distributed Systems, Tianjin, China, 2019, pp. 985-989.

[6] A. Y. S. Lam et al., "Coordinated autonomous vehicle parking for vehicle-to-grid services," in Proc. IEEE International Conference on Smart Grid Communications, Sydney, Australia, 2016 , pp. 284-289.

[7] C. Zhang et al., "FASTGNN: A topological information protected federated learning approach for traffic speed forecasting," IEEE Transactions on Industrial Informatics, vol. 17, no. 12, pp. 8464-8474, 2021.

[8] J. J. Q. Yu et al., "Online false data injection attack detection with wavelet transform and deep neural networks," IEEE Transactions on Industrial Informatics, vol. 14, no. 7, pp. 3271-3280, Jul. 2018.

[9] J. J. Q. Yu, "Sybil attack identification for crowdsourced navigation: a self-supervised deep learning approach," IEEE Transactions on Intelligent Transportation Systems, vol. 22, no. 7, pp. 4622-4634, 2020 .

[10] J. J. Q. Yu et al., "Real-coded chemical reaction optimization with different perturbation functions," in Proc. IEEE Congress of Evolutionary Computation, Brisbane, Australia, 2012, pp. $1-8$.

[11] A. Y. S. Lam et al., "Coordinated autonomous vehicle parking for vehicle-to-grid services: Formulation and distributed algorithm," IEEE Transactions on Smart Grid, vol. 9, no. 5, pp. 4356-4366, Sep. 2018

[12] J. J. Q. Yu et al., "Evolutionary artificial neural network based on chemical reaction optimization," in Proc. IEEE Congress of Evolutionary Computation, New Orleans, LA, US, 2011, pp. 2083-2090.

[13] C. Zhang et al., "Spatial-temporal graph attention networks: a deep learning approach for traffic forecasting," IEEE Access, vol. 7, pp. 166 246-166 256, 2019.

[14] J. J. Q. Yu et al., "Chemical reaction optimization for the set covering problem," in Proc. IEEE Congress of Evolutionary Computation, Beijing, China, 2014, pp. 512-519.

[15] J. J. Q. Yu, "Semi-supervised deep ensemble learning for travel mode identification," Transportation Research Part C: Emerging Technologies, vol. 112, pp. 120-135, Mar. 2020.

[16] _ _ "Two-stage request scheduling for autonomous vehicle logistic system," IEEE Transactions on Intelligent Transportation Systems, vol. 20, no. 5, pp. 1917-1929, May 2019.

[17] J. J. Q. Yu et al., "Autonomous vehicle logistic system: Joint routing and charging strategy," IEEE Transactions on Intelligent Transportation Systems, vol. 19, no. 7, pp. 2175-2187, Jul. 2018

[18] _ _ "A social spider algorithm for solving the non-convex economic load dispatch problem," Neurocomputing, vol. 171, pp. 955-965, Jan. 2016.

[19] _ _ "Base station switching problem for green cellular networks with social spider algorithm," in Proc. IEEE Congress of Evolutionary Computation, Beijing, China, 2014, pp. 2338-2344.

[20] _ - "Intelligent fault detection scheme for microgrids with wavelet-based deep neural networks," IEEE Transactions on Smart Grid, vol. 10, no. 2, pp. 1694-1703, Mar. 2019.

[21] Y. Liu et al., "Privacy-preserving traffic flow prediction: a federated learning approach," IEEE Internet of Things Journal, vol. 7, no. 8, pp. 7751-7763, Aug. 2020.

[22] J. J. Q. Yu et al., "Online vehicle routing with neural combinatorial optimization and deep reinforcement learning," IEEE Transactions on Intelligent Transportation Systems, vol. 20, no. 10 , pp. 3806-3817, Oct. 2019.

[23] _ - "Real-time traffic speed estimation with graph convolutional generative autoencoder," IEEE Transactions on Intelligent Transportation Systems, vol. 20, no. 10, pp. 3940-3951, Oct. 2019. 
[24] A. Y. S. Lam et al., "Power-controlled cognitive radio spectrum allocation with chemical reaction optimization," IEEE Transactions on Wireless Communications, vol. 12, no. 7, pp. 3180-3190, Jul. 2013.

[25] J. J. Q. Yu et al., "Synchrophasor recovery and prediction: a graph-based deep learning approach," IEEE Internet of Things Journal, vol. 6, no. 5, pp. 7348-7359, Oct. 2019.

[26] _ _ "Delay aware intelligent transient stability assessment system," IEEE Access, vol. 5, pp. $17230-17239,2017$.

[27] _ - "Intelligent time-adaptive transient stability assessment system," IEEE Transactions on Power Systems, vol. 33, no. 1, pp. 1049-1058, Jan. 2018.

[28] _ _ , "Double auction-based pricing mechanism for autonomous vehicle public transportation system," IEEE Transactions on Intelligent Vehicles, vol. 3, no. 2, pp. 151-162, Jun. 2018.

[29] —_- "Maximizing aggregator profit through energy trading by coordinated electric vehicle charging," in Proc. IEEE International Conference on Smart Grid Communications, Sydney, Australia, 2016, pp. 497-502.

[30] Y. Zhu et al., "Robust federated learning approach for travel mode identification from nonIID GPS trajectories," in Proc. IEEE International Conference on Parallel and Distributed Systems, Hong Kong, China, 2020, pp. 585-592.

[31] J. J. Q. Yu et al., "Delay aware power system synchrophasor recovery and prediction framework," IEEE Transactions on Smart Grid, vol. 10, no. 4, pp. 3732-3742, Jul. 2019.

[32] —_ "Sensor deployment for air pollution monitoring using public transportation system," in Proc. IEEE Congress of Evolutionary Computation, Brisbane, Australia, 2012, pp. 1-7.

[33] _- "Long-term urban traffic speed prediction with deep learning on graphs," IEEE Transactions on Intelligent Transportation Systems, 2021, in press.

[34] _ _ "A social spider algorithm for global optimization," Applied Soft Computing, vol. 30, pp. 614-627, May 2015.

[35] J. J. Q. Yu, "Citywide traffic speed prediction: a geometric deep learning approach," Knowledge-Based Systems, p. 106592, Nov. 2020. 